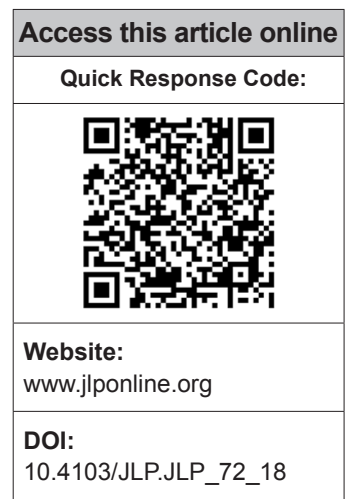

Division of Trauma Surgery and Critical Care, JPNATC, AlIMS, Departments of ${ }^{1}$ Microbiology and ${ }^{3}$ Laboratory Medicine, JPNATC, AIIMS, New Delhi, ${ }^{2}$ Department of Orthopaedics, AllMS, New Delhi, India

Address for correspondence: Prof. Purva Mathur, Room No-211, Second

Floor, Microbiology Lab, JPNATC, AIIMS, New Delhi - 110 029, India.

E-mail: purvamathur@ yahoo.co.in

Submission: 26-05-2018 Accepted: 15-10-2018

\title{
A 5-year surveillance on antimicrobial resistance of Acinetobacter isolates at a level-I trauma centre of India
}

\author{
Minu Kumari, Priyam Batra', Rajesh Malhotra², Purva Mathur ${ }^{3}$
}

\section{Abstract:}

INTRODUCTION: Acinetobacter spp. has emerged as a major cause of nosocomial outbreaks. Multiple antibiotic resistance is an important problem in Acinetobacter isolates in recent years. The aim of this study was to evaluate the rate of antimicrobial resistance and changes in resistance pattern over a period of 5 years (2012-2016) in Acinetobacter spp. isolated from trauma patients.

MATERIALS AND METHODS: Acinetobacter spp. was identified by VITEK 2 and antibiotic susceptibility of isolates was investigated by disc-diffusion method and VITEK 2 automated system. Interpretation of susceptibility results was based on the Clinical and Laboratory Standards Institute guidelines.

RESULTS: Out of the total 16,210 isolates obtained throughout the period of 5 years, Acinetobacter spp. accounted for $3744(28.9 \%)$. Out of which, the species which was maximally isolated was Acinetobacter baumannii (98.5\%), followed by Acinetobacter Iwoffii (1.4\%) and Acinetobacter hemolyticus $(0.1 \%)$. The highest number of clinical isolates of Acinetobacter were recovered from neurosurgical ward $(n=1210)$, followed by the neurosurgical intensive care unit (ICU) $(n=1000)$ and surgical ICU $(n=948)$ and the most common sample of Acinetobacter isolation was from tracheal aspirate $(37.1 \%)$, followed by wound swab (18.8\%). The highest level of resistance was observed against ciprofloxacin (96\%), followed by cefepime (95\%), ceftazidime $(95 \%)$, piperacillin $(95 \%)$, and amikacin (92\%). The trend of antibiotic resistance was found to be statistically significant $(P<0.001)$ for most of the antibiotics being tested such as amikacin and carbapenems.

CONCLUSION: The high rate of antibiotic resistance of the Acinetobacterstrains indicated that there is an urgent need for controlled antibiotic usage and appliance of hospital infection control measures.

Key words:

Antimicrobial, infection, resistance, surveillance

\section{Introduction}

Tnfections due to multidrug-resistant (MDR) Lbacteria are responsible for significant morbidity and mortality. ${ }^{[1]}$ Of these MDR organisms, Acinetobacter spp. remains one of the most dreadful opponents, as its unique and multifarious resistance mechanism allows it to elude the activity of variety of currently known and available drugs. Acinetobacter spp. are aerobic Gram-negative bacteria that do not ferment glucose and are pervasive

This is an open access journal, and articles are distributed under the terms of the Creative Commons Attribution-NonCommercial-ShareAlike 4.0 License, which allows others to remix, tweak, and build upon the work non-commercially, as long as appropriate credit is given and the new creations are licensed under the identical terms.

For reprints contact: reprints@medknow.com in the environment. In hospital settings, these nosocomial pathogens affect severely ill patients and cause a wide spectrum of infections. Acinetobacter spp. alone is responsible for approximately $10.6 \%$ of mortality due to MDR health-care-associated infections, with an estimated cost ranging from US $\$ 33,510$ to $\$ 129,917 .[2,3]$ The infections caused by Acinetobacter spp. are often associated with invasive operative procedures and devices. ${ }^{[4]}$ It has also been implicated in wound infections, endocarditis, peritonitis, ventriculitis, abdominal and urinary tract infections, and meningitis. ${ }^{[4]}$

How to cite this article: Kumari $M$, Batra $P$, Malhotra R, Mathur P. A 5-year surveillance on antimicrobial resistance of Acinetobacter isolates at a level-I trauma centre of India. J Lab Physicians $2019 ; 11: 34-8$ 
Acinetobacter spp. are resistant to many antibiotics because of the low permeability of its outer cell membrane and constitutive expression of certain efflux pumps, and it can accumulate components of resistance mechanisms encoded on plasmids, integrons, and transposons in hospital settings associated with high antibiotic consumption. ${ }^{[5]}$ Besides that, Acinetobacter has the ability to survive for a long period of time on hospital equipments. ${ }^{[6]}$

In recent years, the drug resistance rates of Acinetobacter are increasing worldwide, particularly with the appearance of carbapenem-resistant Acinetobacter. ${ }^{[7]}$ In several studies, infection with MDR Acinetobacter has been associated with increased mortality. ${ }^{[8]}$ The trend in increasing antimicrobial resistance (AMR) limits the choice of effective antimicrobial agents. Thus, continuous surveillance of AMR of Acinetobacter is very important for the selection and implementation of proper empirical therapy for seriously ill patients.

The aim of this study was to determine the antibiotic resistance patterns of Acinetobacter that cause infections in the intensive care units (ICUs) and wards at a Level 1 trauma care center in a developing country and to follow the variation in resistance pattern from 2012 to 2016.

\section{Materials and Methods}

The present study was a retrospective cross-sectional study of all the nonduplicate isolates (i.e., similar isolates obtained over a 2-week period were regarded as one) of Acinetobacter that were collected and processed by the microbiological laboratory in our hospital from January 2012 to December 2016. The study hospital is a 186-bedded, tertiary care, Level 1 trauma center in India. Blood, respiratory tract specimens, urine, pus from wounds, cerebrospinal fluid (CSF), tissue sample, pleural fluid and other biological specimens collected from patients admitted to any unit of the hospital, and follow-up patients were eligible for inclusion. Samples sent by the clinicians in our hospital are generally from symptomatic patients only, and the history of the patients is generally communicated along with the samples. Thus, the processing of the samples was done based on the clinical history such that overreporting of organisms or processing of colonizers can be avoided. All the results were noted from the microbiology database and the resistance pattern was observed and analyzed.

Acinetobacter spp. was identified by VITEK 2 system (BioMerieux, Lyon, France) and antibiotic susceptibility of isolates was investigated by disc-diffusion method and VITEK 2-automated system. Interpretation of susceptibility results was based on the Clinical and Laboratory Standards Institute (CLSI) guidelines. ${ }^{[9]}$ The standardized custom sensitivity panel used in the VITEK 2 included 25 different antimicrobials for susceptibility of all the Gram-negative isolates, but for the present study, only the resistance of Acinetobacter spp. to common anti-Acinetobacter agents was analyzed. The antibiotics tested were amikacin $(30 \mu \mathrm{g})$, cefepime $(30 \mu \mathrm{g})$, ceftazidime $(30 \mu \mathrm{g})$, ciprofloxacin $(5 \mu \mathrm{g})$, gentamicin $(10 \mu \mathrm{g})$, imipenem $(10 \mu \mathrm{g})$, levofloxacin $(5 \mu \mathrm{g})$, meropenem $(10 \mu \mathrm{g})$, piperacillin $(100 \mu \mathrm{g})$, piperacillin/tazobactam $(100 / 10 \mu \mathrm{g})$, trimethoprim/sulfamethoxazole $(1.25 / 23.75 \mu \mathrm{g})$, and tigecycline $(15 \mu \mathrm{g})$.

Resistance of Acinetobacter spp. to individual antimicrobials is presented in absolute numbers and percentages, and was analyzed as per year and hospital unit origin (ICU vs. non-ICU). Since the number of isolates of non-baumannii Acinetobacter spp. was not significant, the trend in the resistance pattern was not studied separately. All the statistical analyses were performed with STATA software (STATA Version 12.1, StataCorp, Texas-77845, USA). $P<0.05$ was considered statistically significant.

\section{Results}

During the period of 5 years, 3744 isolates were collected and identified as Acinetobacter, which represented 28.9\% of all the isolated strains. The mean age of the patients was 34 years, ranging from 1 to 96 years. Male patients (3133, $83.7 \%)$ outnumber female patients $(611,16.3 \%)$ from whom Acinetobacter spp. were isolated. In male patients, the mean age was 34 years with a range of 1-96 years, whereas in female patients, the mean age was found to be 35.3 years with a range of 1-90 years. Acinetobacter spp. were observed in patients of different age groups such as in $<15$ years of age $(229,6 \%), 15-24$ years (902, $24 \%), 25-65$ years $(2473,66 \%)$, and $>65$ years $(140$, $4 \%)$. This study is a part of Department of Science \& Technology (DST)-funded project entitled "Molecular and epidemiological study of beta-lactamase producing Gram negative nosocomial pathogens in India," and ethical clearance was obtained from the Institute Ethics Committee of AIIMS (Ref. No.-IEC/NP-123/2011).

Out of total 3744 isolates, the species which was most commonly isolated was Acinetobacter baumannii (3688, 98.5\%), followed by Acinetobacter lwoffii $(52,1.4 \%)$, and Acinetobacter hemolyticus (4, 0.1\%). Acinetobacter $s p p$. were isolated most commonly from neurosurgery ward $(1,210,32 \%)$, followed by neurosurgical ICU (1000, 27\%), surgical ICU $(948,25 \%)$, surgical ward $(442,12 \%)$, follow-up patients $(65,2 \%)$, orthopedic ward $(41,1 \%)$, and casualty (38, 1\%). A. hemolyticus (4) were isolated from the follow-up patients. A. lwoffii (52) were isolated from the surgical ward (18), orthopedic ward (17), casualty (12), and neurosurgical ICU (5). A. 
baumannii were isolated from neurosurgical ICU (9995), surgical ICU (948), surgical ward (424), follow-up patients (61), orthopedic ward (24), and casualty (26).

The Acinetobacter strains were maximally isolated from tracheal aspirate $(1,391,37.1 \%)$, followed by pus / wound swab $(704,18.8 \%)$, bronchoalveolar lavage (BAL) $(612,16.3 \%)$, blood $(502,13.4 \%)$, CSF $(185,4.9 \%)$, urine $(100,2.7 \%)$, central venous catheter tip $(63,1.7 \%)$, tissue sample $(55,1.5 \%)$, drain fluid $(52,1.4 \%)$, pleural fluid $(23,0.6 \%)$, sputum $(4,0.1 \%)$, bone fragments $(2,0.1 \%)$, and others (e.g., - ascites fluid, lavage fluid, debridement slough, etc.) $(51,1.4 \%)$. The resistance pattern of the Acinetobacter spp. (A. baumannii, A. lwoffii, and A. hemolyticus) isolated during the 5 -year study period is described in Table 1. The analysis of the level of antibiotic resistance showed that resistance was maximum against ciprofloxacin $(96 \%)$, followed by ceftazidime (95\%), cefepime (95\%), and piperacillin (95\%). The lowest level of resistance was observed against tigecycline ( $8 \%)$. Of the strains isolated, a total of $68(1.8 \%)$ isolates only were found to be colistin resistant using the VITEK 2 system. Antibiotic resistance pattern of different antimicrobials by specimen origin (ICU vs. non-ICU) for Acinetobacter spp. is shown in Figure 1. The difference in the level of resistance was not found to be statistically significant for any antibiotic tested.

\section{Discussion}

Acinetobacter spp. has emerged as one of the major causes of hospital-acquired infections in critically ill patients. ${ }^{[4]}$ The drug resistance data during 2012-2016 in our hospital setup showed that the resistance rates of the majority of antibiotics increased throughout the period. The antibiotic resistance in our hospital has increased for all antibiotics from 2012 to 2016 by almost the same percentage. However, the increase in

Table 1: Resistance pattern of Acinetobacter spp. against different antimicrobials

\begin{tabular}{lcc|c|c|ccc}
\hline Antimicrobials & $2012(\%)$ & $2013(\%)$ & $2014(\%)$ & $2015(\%)$ & $2016(\%)$ & Total resistance (\%) & Total sensitive (\%) \\
\hline Amikacin & $965(96.8)$ & $1015(93.1)$ & $884(94)$ & $718(90.1)$ & $723(84.5)$ & $4305(92)$ & $375(8)$ \\
Cefepime & $955(96)$ & $1061(97.3)$ & $888(94.5)$ & $765(96)$ & $773(90.3)$ & $4442(95)$ & $238(5)$ \\
Ceftazidime & $975(97.8)$ & $1065(97.7)$ & $878(93.4)$ & $752(94.4)$ & $775(90.5)$ & $4445(95)$ & $235(5)$ \\
Ciprofloxacin & $981(98.4)$ & $1040(95.4)$ & $911(97)$ & $777(97.5)$ & $772(90.2)$ & $4481(96)$ & $199(4)$ \\
Gentamycin & $946(94.9)$ & $1022(93.7)$ & $889(94.6)$ & $749(94)$ & $711(83)$ & $4317(92)$ & $363(8)$ \\
Imipenem & $892(89.5)$ & $954(87.5)$ & $862(91.7)$ & $751(94.2)$ & $759(88.7)$ & $4218(90)$ & $462(10)$ \\
Levofloxacin & $890(89.3)$ & $831(76.2)$ & $661(70.3)$ & $666(83.6)$ & $701(81.9)$ & $3749(80)$ & $931(20)$ \\
Meropenem & $907(91)$ & $953(87.4)$ & $850(90.4)$ & $744(93.4)$ & $759(88.7)$ & $4213(90)$ & $467(10)$ \\
Piperacillin & $961(96.4)$ & $1053(96.6)$ & $901(95.8)$ & $780(97.9)$ & $774(90.4)$ & $4469(95)$ & $211(5)$ \\
Piperacillin/tazobactam & $944(94.7)$ & $1018(93.4)$ & $870(92.5)$ & $744(93.4)$ & $762(89)$ & $4338(93)$ & $342(7)$ \\
Trimethoprim/sulfamethoxazole & $688(69.0)$ & $951(87.2)$ & $798(84.9)$ & $710(89.1)$ & $605(70.75)$ & $3752(80)$ & $928(20)$ \\
Tigecycline & N/A & $85(7.8)$ & $45(4.8)$ & $61(7.7)$ & $89(10.4)$ & $280(8)$ & $3403(92)$ \\
\hline
\end{tabular}

$\mathrm{N} / \mathrm{A}=$ Not available

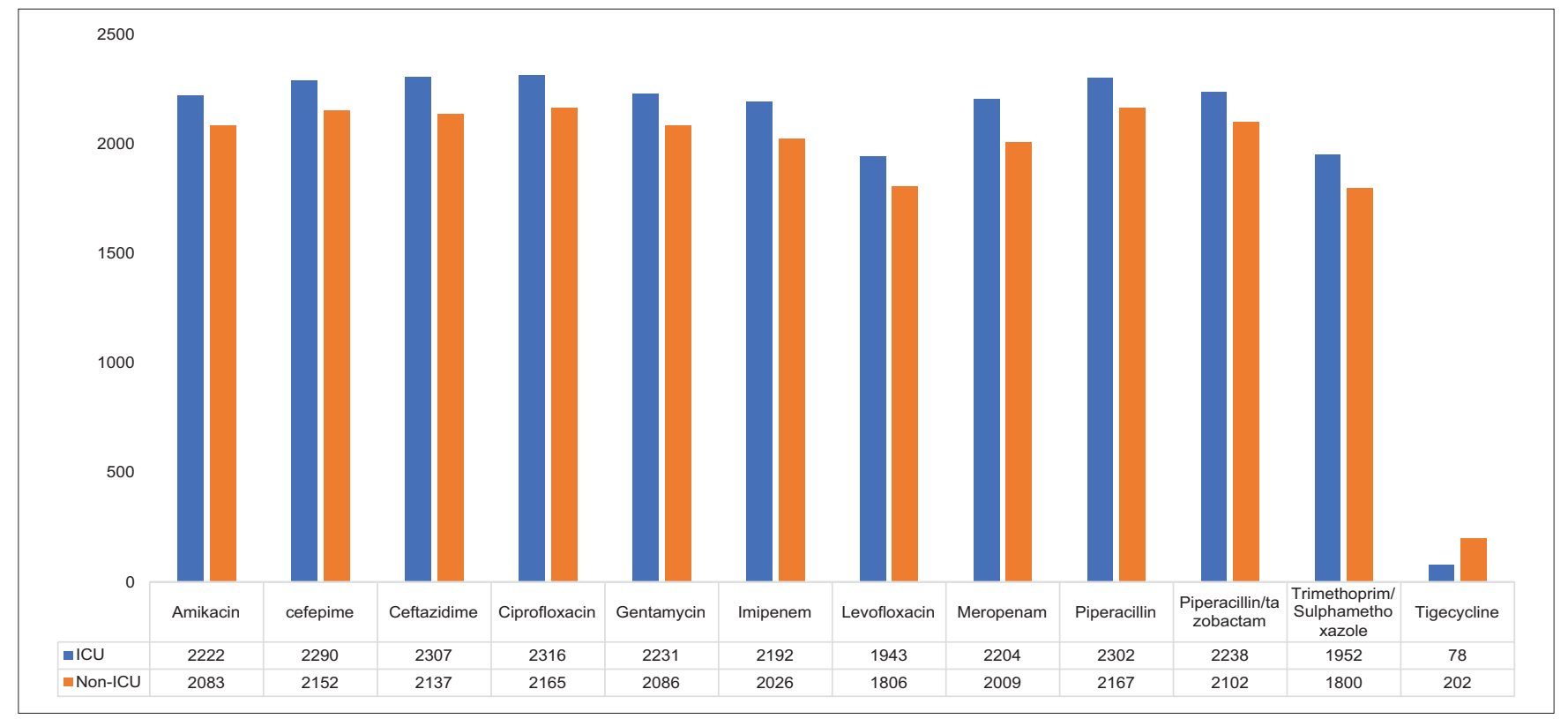

Figure 1: Resistance pattern of different antimicrobials in the intensive care unit versus nonintensive care unit setup throughout the period of 5 years 
the level was maximum for amikacin. The antibiotic policy was introduced in our hospital setting in 2006, and the majority of antibiotics used were restricted and guided by rules. The antibiotic policy promoted the rational use of antimicrobial drugs, and certain antibiotic-resistant rates periodically reduced. However, all the antimicrobial-resistant rates did not decrease at the same time to cure infection, and there was a fluctuation. Rates of some antibiotics periodically increased also in our hospital settings.

Carbapenemase-resistant Acinetobacter is an increasing problem worldwide. ${ }^{[10]}$ In a study in Greece, the evolution of Acinetobacter isolates from carbapenem susceptible in the 1990s to carbapenem resistant in the 2000s was rapid. Resistance to imipenem in Acinetobacter isolates in hospitalized ICU patients increased from 0\% in 1996 to $91 \%$ in 2006 of a Greek hospital. ${ }^{[11]}$ In our study also, we observed the proportion of carbapenem-resistant clinical isolates to be $80 \%-96 \%$. In our hospital setup, $83.7 \%$ of patients infected with Acinetobacter isolates were males which shows the general trend of higher male preponderance in trauma centers. ${ }^{[12]}$

Most of the studies have reported the predominance of Acinetobacter strains in bronchopulmonary samples. ${ }^{[13]}$ In this study also, the main isolation site of the Acinetobacter isolates was from tracheal sample $(37.1 \%)$, followed by BAL (18.8\%) followed by blood cultures (13.4\%). It has been reported that among Acinetobacter species, A. baumannii is the main cause of Acinetobacter infections with the antibiotic resistance rate being very high causing more serious infections than other species of Acinetobacter ${ }^{[14]}$ We have also found the same results in our study as the species which was maximally isolated was A. baumannii (98.5\%).

In general, the Acinetobacter isolates are known for their resistance to various antibiotics despite their weak virulence, limiting the control and treatment due to these microorganisms. ${ }^{[15]}$ Our study shows that the rate of antibiotic resistance in our trauma care center is generally high and variable. Several authors have confirmed the high prevalence of these infections associated with high resistance in ICUs. ${ }^{[16]}$ The high proportion and the high resistance of these microorganisms in ICUs are related to the existence of numerous risk factors associated with Acinetobacter infection such as immunocompromised patients, longer hospital stay, use of invasive devices, the broad-spectrum antibiotics therapy, possible and frequent contaminations, cross transmission of this bacteria through environmental reservoirs, and contaminated hands of health-care workers. ${ }^{[17]}$

For the beta-lactam antibiotics which are a large family playing an important role in antimicrobial treatment, ${ }^{[18]}$ the high resistance of Acinetobacter clinical isolates to this class of antibiotics (ceftazidime, cefepime, imipenem, and piperacillin/tazobactam) has been described in the literature. ${ }^{[19]}$ In our study, the resistance rate was highest in ciprofloxacin, followed by ceftazidime, cefepime, and piperacillin. In our study, $1.8 \%$ of the isolates were found to be colistin resistant using the VITEK 2 system. These strains, however, could not be retested using the currently recommended microbroth dilution method. However, a major drawback of our study is that we did not test for the susceptibility to antibiotics such as ampicillin/sulbactam, minocycline, cefoperazone/sulbactam, or cefepime/tazobactam which have proved to have promising effect in the coming era of widespread antibiotic resistance.

\section{Conclusion}

The vast majority of Acinetobacter spp. in our hospital are MDR. The remaining therapeutic options for Acinetobacter infections are severely limited. Continuous surveillance of AMR of Acinetobacter sp. is extremely important for the selection of appropriate empirical therapy. Infection control practices and antimicrobial stewardship should be implemented in every hospital setting. The resistance pattern shown in the study could be used for updating the antibiotic policy of our institute.

\section{Financial support and sponsorship}

Nil.

\section{Conflicts of interest}

There are no conflicts of interest.

\section{References}

1. Centers for Disease Control and Prevention. Antibiotic Resistance Threats in the United States; 2013. Available from: http:/ / www.cdc.gov/drugresistance/threat-report-2013/pdf/ ar-threats-2013-508.pdf. [Last accessed on 2018 May 02].

2. Magiorakos AP, Srinivasan A, Carey RB, Carmeli Y, Falagas ME, Giske CG, et al. Multidrug-resistant, extensively drug-resistant and pandrug-resistant bacteria: An international expert proposal for interim standard definitions for acquired resistance. Clin Microbiol Infect 2012;18:268-81.

3. Nelson RE, Schweizer ML, Perencevich EN, Nelson SD, Khader K, Chiang HY, et al. Costs and mortality associated with multidrug-resistant healthcare-associated Acinetobacter infections. Infect Control Hosp Epidemiol 2016;37:1212-8.

4. Antunes LC, Visca P, Towner KJ. Acinetobacter baumannii: Evolution of a global pathogen. Pathog Dis 2014;71:292-301.

5. Maragakis LL, Perl TM. Acinetobacter baumannii: Epidemiology, antimicrobial resistance, and treatment options. Clin Infect Dis 2008;46:1254-63.

6. Liu WL, Liang HW, Lee MF, Lin HL, Lin YH, Chen CC, et al. The impact of inadequate terminal disinfection on an outbreak of imipenem-resistant Acinetobacter baumannii in an Intensive Care Unit. PLoS One 2014;9:e107975.

7. Souli M, Galani I, Giamarellou H. Emergence of extensively 
drug-resistant and pandrug-resistant Gram-negative bacilli in Europe. Euro Surveill 2008;13. pii: 19045.

8. Kwon KT, Oh WS, Song JH, Chang HH, Jung SI, Kim SW, et al. Impact of imipenem resistance on mortality in patients with Acinetobacter bacteraemia. J Antimicrob Chemother 2007;59:525-30.

9. Clinical and Laboratory Standard Institutes (CLSI). Performance Standards for Antimicrobial Susceptibility Testing. CLSI Supplement M100. $27^{\text {th }}$ ed. Wayne PA: Clinical and Laboratory Standard Institutes; 2017.

10. Falagas ME, Mourtzoukou EG, Polemis M, Vatopoulos AC; Greek System for Surveillance of Antimicrobial Resistance. Trends in antimicrobial resistance of Acinetobacter baumannii clinical isolates from hospitalised patients in Greece and treatment implications. Clin Microbiol Infect 2007;13:816-9.

11. García-Garmendia JL, Ortiz-Leyba C, Garnacho-Montero J, Jiménez-Jiménez FJ, Pérez-Paredes C, Barrero-Almodóvar $\mathrm{AE}$, et al. Risk factors for Acinetobacter baumannii nosocomial bacteremia in critically ill patients: A cohort study. Clin Infect Dis 2001;33:939-46.

12. Punpanich W, Nithitamsakun N, Treeratweeraphong V, Suntarattiwong P. Risk factors for carbapenem non-susceptibility and mortality in Acinetobacter baumannii bacteremia in children. Int J Infect Dis 2012;16:e811-5.

13. Jaggi N, Sissodia P, Sharma L. Acinetobacter baumannii isolates in a tertiary care hospital: Antimicrobial resistance and clinical significance. J Microbiol Infect Dis 2012;2:57-63.

14. Nazmul MH, Jamal H, Fazlul MK. Acinetobacter species-associated infections and their antibiotic susceptibility profiles in Malaysia. Biomed Res India 2012;23:571-5.

15. Chuang YC, Sheng WH, Li SY, Lin YC, Wang JT, Chen YC, et al. Influence of genospecies of Acinetobacter baumannii complex on clinical outcomes of patients with Acinetobacter bacteremia. Clin Infect Dis 2011;52:352-60.

16. Obeidat N, Jawdat F, Al-Bakri AG, Shehabi AA. Major biologic characteristics of Acinetobacter baumannii isolates from hospital environmental and patients' respiratory tract sources. Am J Infect Control 2014;42:401-4.

17. Xu J, Sun Z, Li Y, Zhou Q. Surveillance and correlation of antibiotic consumption and resistance of Acinetobacter baumannii complex in a tertiary care hospital in Northeast China, 2003-2011. Int J Environ Res Public Health 2013;10:1462-73.

18. Özgür ES, Horasan ES, Karaca K, Ersöz G, Naycı Atış S, Kaya A. Ventilator-associated pneumonia due to extensive drug-resistant Acinetobacter baumannii: Risk factors, clinical features, and outcomes. Am J Infect Control 2014;42:206-8.

19. Queenan AM, Pillar CM, Deane J, Sahm DF, Lynch AS, Flamm RK, et al. Multidrug resistance among Acinetobacter spp. in the USA and activity profile of key agents: Results from CAPITAL surveillance 2010. Diagn Microbiol Infect Dis 2012;73:267-70. 